\title{
LXVI. ON THE PHOTO-OXIDATION OF ADRENALINE.
}

\author{
By TOMÁ̆ VACEK'. \\ From the Department of Physiology and Biochemistry, \\ University College, London.
}

(Received January 18th, 192\%.)

In preliminary experiments it was observed that the presence of a very small amount of adrenaline caused blood-plasma to absorb in ultra-violet light about four times as much oxygen as the plasma alone. It was therefore decided to test the behaviour of adrenaline in ultra-violet light, employing the technique devised by Harris [1926]. Two quartz tubes, one for the adrenaline and the other the control, coupled to a Haldane volumeter were irradiated with a mercury-vapour lamp $\left(\frac{1}{2} \mathrm{kw}\right.$.) at a distance of $15 \mathrm{~cm}$. The tubes were kept in a bath at $37^{\circ}$. Different preparations of adrenaline were used: adrenaline hydrochloride in solution (Parke, Davis) and pure adrenaline (Parke, Davis), dissolved either in distilled water or in $0.5 \%$ hydrochloric acid. Amounts of 0.0005 to $0.004 \mathrm{~g}$. adrenaline dissolved in $45 \mathrm{cc}$. distilled water were used.

In the first place it was determined whether oxygen absorption takes place in the dark. The volume in the adrenaline tube remained unchanged when 0.0005 to $0.004 \mathrm{~g}$. adrenaline was used. Pure adrenaline, dissolved in distilled water, only showed occasionally a minute uptake of oxygen. The solution became more or less pink after a long exposure to ultra-violet radiation. The intensity of the pink colour is in direct relation to the amount of oxygen absorbed by the adrenaline.

The oxygen uptake by different amounts of adrenaline was next determined. The adrenaline hydrochloride solution in volumes from $0.5 \mathrm{cc}$. to $4 \mathrm{cc}$. was used; this solution is called A. The results are shown in Fig. 1. The higher the concentration of the adrenaline the greater the absorption of the oxygen. But the amount of the oxygen is not in direct proportion to the amount of adrenaline. The oxygen uptake by adrenaline of a higher concentration is relatively less. It is, however, probable that the absorption would be greater after a longer irradiation, and if the partial pressure of oxygen in the tubes were maintained at the same level throughout the experiments.

In the next experiments the different intensities of oxidation of different kinds of adrenaline under various conditions were tested. Here $0.001 \mathrm{~g}$. of adrenaline was used and irradiated for 30 minutes. Pure adrenaline, dissolved in distilled water (C), has a very intense oxidation (Fig. 2).

1 Fellow of the International Education Board. 
It was further shown that hydrochloric acid is a good protective against photo-oxidation even in very low concentration ( $0.5 \%$ ). After an irradiation of 30 minutes adrenaline $(C)$ took up six times more oxygen than adrenaline dissolved in $0.5 \% \mathrm{HCl}(\mathrm{B})$. And even after switching off the light the oxidation of the adrenaline $\mathrm{C}$ continued, though not so intensely (Fig. 2).

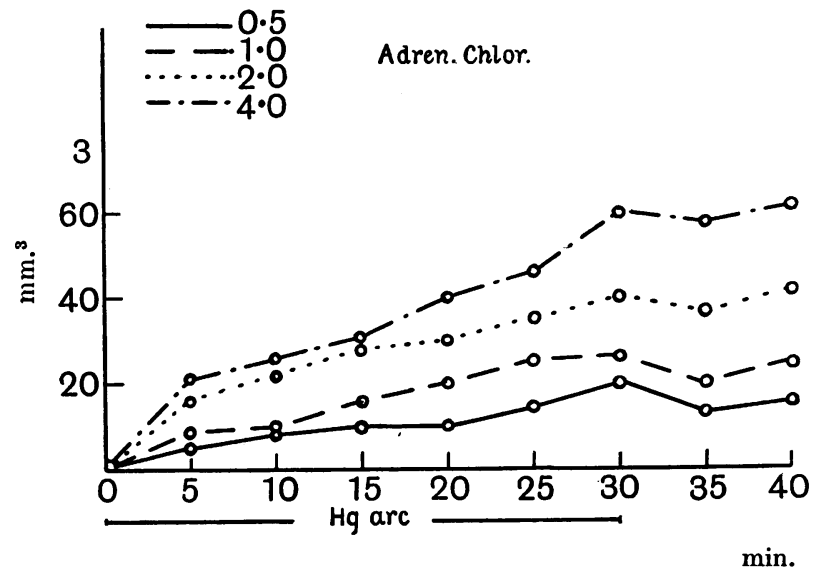

Fig. 1.

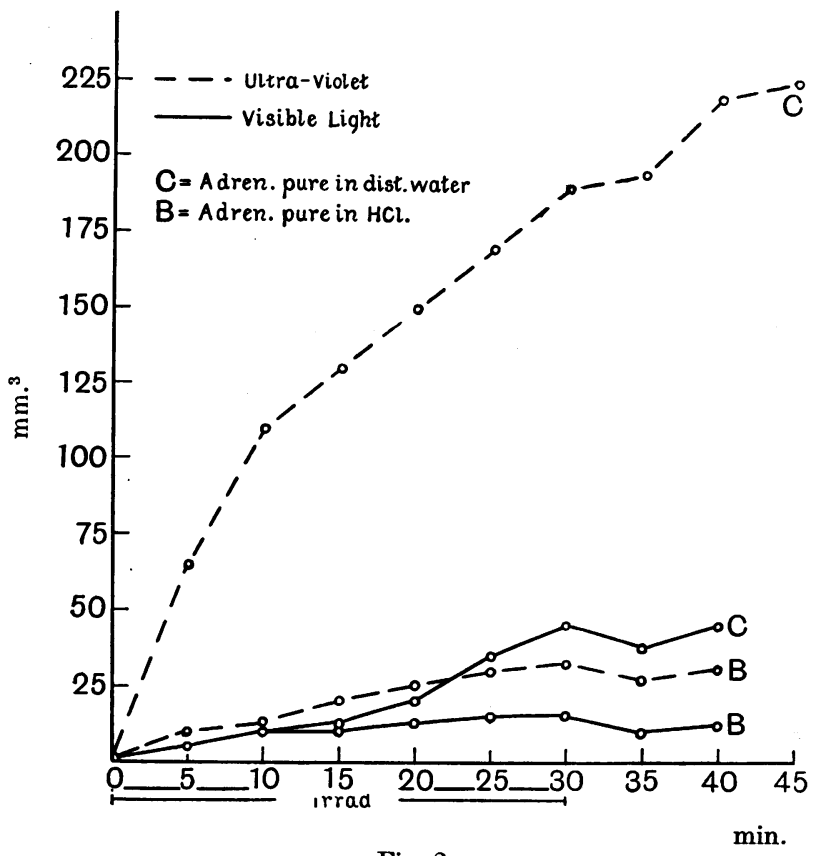

Fig. 2.

It was necessary to examine the behaviour of hydrochloric acid itself in the ultra-violet radiation. It was found that neither distilled water nor the 
amount of $\mathrm{HCl}$ here used $(1 \mathrm{cc} .0 .5 \% \mathrm{HCl}$ ) absorbed any appreciable amount of oxygen after a $30 \mathrm{~min}$. exposure to the radiation of a mercury-vapour lamp.

It is well known that adrenaline oxidises in daylight and in these investigations it was also the object to determine the degree of oxidation in an intense light but without ultra-violet rays. In order to filter the short-wave rays a triple plate-glass filter was put between the source of the radiant energy and the quartz tubes. The two solutions (B, C) of adrenaline ( $(0.002 \mathrm{~g}$.) were examined and the results are given in Fig. 2. An oxidation took place even in the visible light, though it was much less than that caused by ultra-violet rays. The greatest uptake of oxygen in the presence of visible light only was observed in the adrenaline solution (C), whilst (B) seems to be very resistant to the influence of visible light.

\section{Conclusions.}

Different preparations of adrenaline show different tendencies to oxidation. Ultra-violet radiation causes an acceleration of the oxidation of adrenaline even in very large dilutions. The greatest sensitivity to light is shown by pure adrenaline in distilled water. Adrenaline hydrochloride solution shows a very low degree of oxidation.

Adrenaline exposed to visible light only behaves similarly, but the oxidation is less intense than when exposed to the short-wave rays.

The pink colour developed by adrenaline when exposed to the ultra-violet radiations is related to the uptake of oxygen. The more oxygen absorbed by the adrenaline the more pink it becomes. It would be interesting to examine the physiological action of this adrenaline, changed by the absorption of oxygen. In the paper of Azuma and Hill [1926] there appeared a short note that adrenaline, after exposure to the light, becomes inactive.

In experiments in which adrenaline from tablets (Parke, Davis, and Burroughs, Wellcome) was used, the oxygen uptake was very intense, but it is uncertain whether this may not be caused by unknown admixtures.

\section{REFERENCES.}

Azuma and Hill (1926). Proc. Roy. Soc. Lond. B, 99, 221.

Harris (1926). Biochem. J. 20, 271. 